\title{
Self-organized criticality in structured neural networks
}

\author{
Maximilian Uhlig ${ }^{1,2^{*}}$, Anna Levina ${ }^{1,3}$, Theo Geisel ${ }^{1,2}$, Michael J Herrmann ${ }^{1,4}$ \\ From Twenty Second Annual Computational Neuroscience Meeting: CNS*2013 \\ Paris, France. 13-18 July 2013
}

Critical dynamics in neural networks is an experimentally and conceptually established phenomenon which has been shown to be important for information processing in the brain. Critical neural networks have been shown to have optimal computational capabilities, information transmission and capacity $[1,2]$. At the same time the theoretical understanding of neural avalanches has been developed starting from sandpile-like system and homogeneous networks towards structured networks. The network connectivity has been chosen, however, as to support or even to enable criticality. There are, nevertheless, many influences that shape the connectivity structure and weighting. Most prominently, this includes Hebbian learning and homeostatic effects, but also pathological changes.
We study how the structural changes affect the presence of criticality in the networks. While homeostatic plasticity may well have a regulatory effect that supports criticality, this cannot been said about Hebbian learning which essentially imprints structure from internally or externally caused activation patterns in the synaptic weighting of the network increasing thus the probability of previous patterns to reoccur. Unless the patterns are carefully chosen to produce critical behavior, these effects have a tendency to counteract critical behavior, e. g. by introducing a particular scale that corrupts the power-law distributions characteristic for critical behavior. Little is known, in particular, about the influence of criticality on associative memory neural networks.
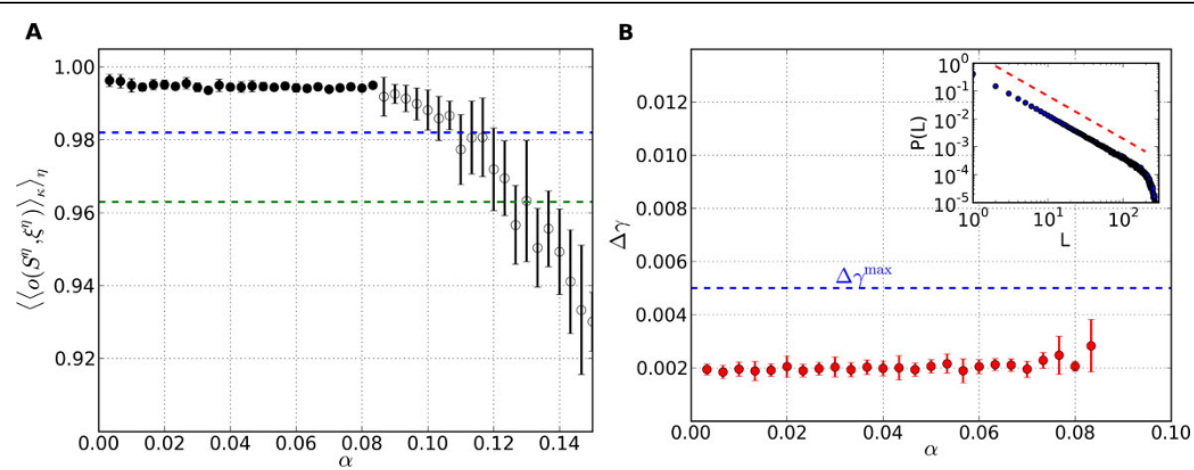

Figure 1 A: Retrieval performance for networks with dynamical synapses and subject to Hebbian learning for different load parameters $\boldsymbol{\alpha}$. Shown is the average overlap between stored patterns and the corresponding retrieved patterns. Dashed lines indicate overlaps corresponding to an average distortion by two digits (lower line) and one digit (upper line). B: Average mean-squared deviation $\Delta \gamma$ from the best-fit power law. All data points lie below the threshold of $\Delta \gamma=0.005$ for critical distribution. The inset shows an example avalanche size distribution $P(L)$ in the converged state and the red dashed line marks the slope of the best-fit power law.

\footnotetext{
* Correspondence: j.michael.herrmann@gmail.com

'Bernstein Center for Computational Neuroscience Göttingen, 37077,

Germany

Full list of author information is available at the end of the article
} 
We found that the critical regime is can be stabilized by short-term synaptic dynamics in the form of synaptic depression and facilitation that was already shown to play an important role in the self-organization of critical neural dynamics [3] or, alternatively, by homeostatic adaptation of the synaptic weights. We show that a heterogeneous distribution of maximal synaptic strengths does not preclude criticality if the Hebbian learning is alternated with periods of critical dynamics recovery.

\section{Acknowledgements}

Supported by the Federal Ministry of Education and Research (BMBF) Germany under grant number 01GQ1005B.

\section{Author details}

${ }^{1}$ Bernstein Center for Computational Neuroscience Göttingen, 37077 ,

Germany. ${ }^{2}$ MPI for Dynamics and Self-Organization, Am Faßberg 17, 37077

Göttingen, Germany. ${ }^{3}$ Max-Planck-Institute for Mathematics in the Sciences,

Leipzig, 04103, Germany. ${ }^{4}$ University of Edinburgh, IPAB, School of

Informatics, Edinburgh EH8 9AB, U.K.

Published: 8 July 2013

\section{References}

1. Beggs J, Plenz D: Neuronal avalanches in neocortical circuits. J Neurosci 2003, 23:11167-11177.

2. Shew WL, Plenz D: The functional benefits of criticality in the cortex. Neuroscientist 2013, 19:88-100.

3. Levina A, Herrmann JM, Geisel T: Dynamical synapses causing selforganized criticality in neural networks. Nat Phys 2007, 3:857-860.

doi:10.1186/1471-2202-14-S1-P168

Cite this article as: Uhlig et al:: Self-organized criticality in structured neural networks. BMC Neuroscience 2013 14(Suppl 1):P168.

\section{Submit your next manuscript to BioMed Central} and take full advantage of:

- Convenient online submission

- Thorough peer review

- No space constraints or color figure charges

- Immediate publication on acceptance

- Inclusion in PubMed, CAS, Scopus and Google Scholar

- Research which is freely available for redistribution

Submit your manuscript at www.biomedcentral.com/submit 